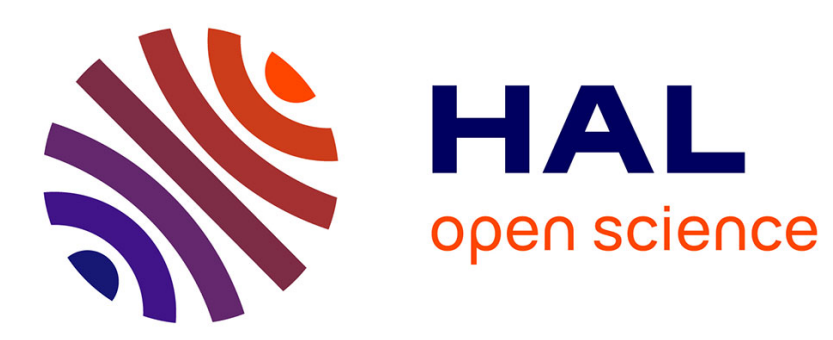

\title{
Analysis of a periodic optimal control problem connected to microalgae anaerobic digestion
}

Térence Bayen, Francis Mairet, Pierre Martinon, Matthieu Sebbah

\section{To cite this version:}

Térence Bayen, Francis Mairet, Pierre Martinon, Matthieu Sebbah. Analysis of a periodic optimal control problem connected to microalgae anaerobic digestion. Optimal Control Applications and Methods, 2015, 36 (6), pp.750-773. 10.1002/oca.2127 . hal-00860570

\section{HAL Id: hal-00860570 https://hal.science/hal-00860570}

Submitted on 12 Sep 2013

HAL is a multi-disciplinary open access archive for the deposit and dissemination of scientific research documents, whether they are published or not. The documents may come from teaching and research institutions in France or abroad, or from public or private research centers.
L'archive ouverte pluridisciplinaire HAL, est destinée au dépôt et à la diffusion de documents scientifiques de niveau recherche, publiés ou non, émanant des établissements d'enseignement et de recherche français ou étrangers, des laboratoires publics ou privés. 


\title{
Optimizing the anaerobic digestion of microalgae in a coupled process
}

\author{
Terence Bayen*, Francis Mairet ${ }^{\dagger}$, Pierre Martinon $^{\ddagger}$, Matthieu Sebbah $^{\S}$
}

September 10, 2013

\begin{abstract}
This work is devoted to the study of an optimal control problem governed by a system describing the coupling of a culture of micro-algae limited by light and an anaerobic digester. The mathematical model for the dynamics of the reactors takes into account a periodic day-night model of the light in the culture of micro-algae and a chemostat model for the digester. Our aim is to optimize the production of methane in the digester during a certain number of days with respect to the dilution rate (the input flow of micro-algae in the digester). We first prove existence and attraction of periodic solutions of the problem for a one day period and we apply Pontryagin Maximum Principle (PMP) in order to characterize optimal controls. We provide numerical simulations of the problem for different light models by a direct method that we refine using an indirect shooting method based on the PMP. We also investigate the dependence of the optimal cost with respect to the ratio of the volumes of the two tanks. Finally, the mean value of the optimal cost over a large number of days without periodic constraints is compared to the optimal cost over one period.
\end{abstract}

\section{Introduction}

Microalgae are cultivated nowadays for feed, food, or cosmetics [1] and they have recently emerged as an attractive alternative for sustainable energy production [2]. Anaerobic digestion can be applied to recover the energy stored in the microalgae biomass by converting it into biogas [3]. This process also leads to ammonium and phosphate release, which can in turn be source of nutrients for the microalgae culture. Coupling microalgae culture and anaerobic digestion is therefore a promising approach to convert solar energy into methane. However, due to their inherent complexity, the control and optimization of such coupled systems are challenging.

We consider a system with a first reactor for microalgae culture and a second one where microalgae (serving as input substrate) are digested and converted into biogas. One essential feature of the model is that the evolution of the concentration of microalgae in the first reactor is limited by light. We assume the light intensity to be a positive function during the day and zero during night, and study several models: a piecewise constant light model (see [4]), continuous and $C^{1}$ regularizations of this first model, and then an oscillating model that better fits the light distribution during the day. The digestion process in the second reactor is modeled as a chemostat with one species and one substrate, see [5].

The main axis of this paper is to study the optimal production of methane in the system over a large timeframe. A first approach is to find an optimal feeding strategy for one day under periodic constraints. Such a policy has the practical advantage that it can be repeated day after day, see e.g. [6]. However, optimizing the system directly over a large timeframe without imposing periodic constraints could lead to a different optimal strategy with a greater production of biogas. One objective of this work is to study precisely this point.

The model is described by a three-dimensional non-autonomous ODE system with periodic constraints, which is linear with respect to the control. Characterizing the optimal solution in this setting is usually quite

\footnotetext{
*Université Montpellier 2, CC 051, 34095 Montpellier cedex 5 and INRA-INRIA 'MODEMIC' team, INRIA Sophia-Antipolis Méditerranée, UMR INRA-SupAgro 729 'MISTEA' 2 place Viala 34060 Montpellier, France tbayen@math.univ-montp2.fr

${ }^{\dagger}$ Inria BIOCORE, BP93, 06902 Sophia-Antipolis Cedex, France. francis.mairet@inria.fr

${ }^{\ddagger}$ INRIA Saclay , Ecole Polytechnique, CMAP 91128 Palaiseau, France. pierre.martinon@inria.fr

§INRA-INRIA 'MODEMIC' team, INRIA Sophia-Antipolis Méditerranée, UMR INRA-SupAgro 729 'MISTEA' 2 place Viala 34060 Montpellier, France matthieu.sebbah@inria.fr
} 
difficult. Using the Maximum Principle, we see that optimal control strategies are a concatenation of bang arcs (i.e the control is extremal) and singular arcs. Singular arcs are important from a practical point of view as they can be computed in feedback form, see [7, 8, 9].

The numerical simulations presented in this paper use both direct and indirect methods, with the softwares Bocop[10] and Sноот[11]. The direct method uses a time discretization to transform the infinite-dimensional optimal control problem into a nonlinear optimization problem, solved here by interior point techniques. The indirect method uses a shooting (with a quasi-Newton algorithm) to solve the Boundary Value Problem arising from the Maximum Principle. Indirect methods are extremely fast and can provide very accurate solutions, but are usually difficult to initialize properly. In this work, we used the solution from the direct method to obtain a suitable initial guess.

The first section of the paper presents the model and the optimization problem. The second section states the results on the existence and attraction of periodic solutions, using the theory of Kolmogorov periodic equations see e.g. $[12,13]$. We also give some characterization of the set of admissible initial conditions for which there exists a periodic solution of the system. In the third section, we apply the Maximum Principle and derive the expression of the singular control (used in the indirect method). Section four is devoted to the numerical simulations, with the impact of the light model on the singular control structure, the optimization of a periodic 1-day cycle, optimization over larger timeframes, and optimization of the volume ratio of the two reactors. Finally, the appendix contains proof of technical results.

\section{Model and statement of the optimal control problem}

\subsection{Model for the coupled digestion of microalgae}

We consider a system of two coupled bioreactors, one for microalgae growth and one for the anaerobic digestion that produces methane, as shown on Fig. 1.

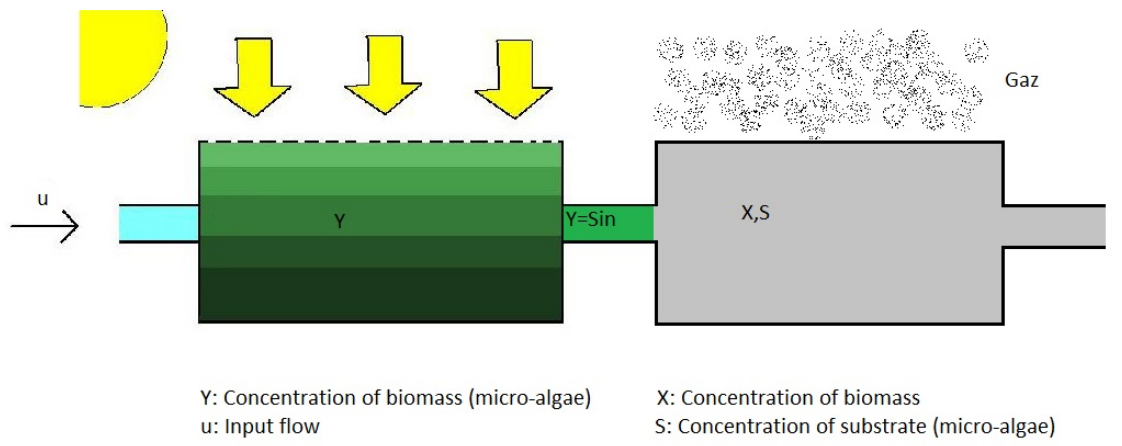

Figure 1: Coupled bioreactors for microalgae growth and anaerobic digestion. Microalgae $y$ serve as input substrate in the second reactor.

We begin with describing the growth of microalgae in the first reactor. We use a similar framework as in [4], and assume that the specific growth rate is a decreasing function of microalgae (because of self-shading), with a constant respiration rate. The simplified dynamics of microalgae $y$ writes:

$$
\frac{d y}{d t}=\frac{\nu(t) y}{\kappa+y}-\frac{Q_{1}}{V_{1}} y-\rho y
$$

where $\kappa>0$ and $\nu(t) \geq 0$ represent the periodic light forcing, $\rho$ is the respiration rate, $V_{1}$ is the volume of the first reactor and $Q_{1} \in\left[0, Q_{1}^{\max }\right]$ is the input flow. Let $T>0$ denote the period corresponding to one day-night cycle, and $\bar{T}=\frac{T}{2}$. For all time $t>0$ such that $t \in[m T+\bar{T},(m+1) T]$ with $m \in \mathbb{Z}$, we take $\nu(t)=0$ (i.e no light during the nights). 
The output flow of the microalgae culture is concentrated and sent in a second reactor, the anaerobic digester. We use a classical bioreactor model (see e.g. [14]) in which the substrate (here the microalgae) is degraded by the biomass according to

$$
\left\{\begin{array}{l}
\frac{d s}{d t}=-k \mu_{2}(s) x+\frac{Q_{2}}{V_{2}}(\alpha y-s), \\
\frac{d x}{d t}=\left(\mu_{2}(s)-\frac{Q_{2}}{V_{2}}\right) x
\end{array}\right.
$$

with $\alpha>0$ a concentration coefficient, $k>0$ a yield coefficient, $x(t)$ the concentration of biomass, $s(t)$ the concentration of substrate, $Q_{2}=Q_{1} / \alpha$ the input flow in the second reactor, $V_{2}$ the volume of this reactor, and $\mu_{2}$ the growth function for the biomass. Gathering the two processes together yields the system:

$$
\left\{\begin{array}{l}
\frac{d y}{d t}=\frac{\nu(t) y}{\kappa+y}-\frac{Q_{1}}{V_{1}} y-\rho y, \\
\frac{d s}{d t}=-k \mu_{2}(s) x+\frac{Q_{2}}{V_{2}}(\alpha y-s), \\
\frac{d x}{d t}=\left(\mu_{2}(s)-\frac{Q_{2}}{V_{2}}\right) x
\end{array}\right.
$$

For convenience, we perform the following change of variable: $(t, y, s, x) \leftarrow\left(\frac{Q_{1}^{\max }}{V_{1}} t, \frac{y}{\kappa}, \frac{s}{k}, x\right)$, and we set the control function $u:=\frac{Q_{1}}{Q_{1}^{\text {max }}}$. The periodic forcing light $\nu$ is then changed into $\mu$ defined by

$$
\mu(t):=\frac{V_{1}}{Q_{1}^{\max }} \nu\left(\frac{V_{1} t}{Q_{1}^{\max }}\right)
$$

In the particular case where $\nu$ is constant over $[0, \bar{T}]$ and zero over $[\bar{T}, T]$, we find that $\mu(t)=\mu_{0}(t)$ where

$$
\mu_{0}(t):= \begin{cases}\bar{\mu}, & t \in[0, \bar{T}], \\ 0, & t \in(\bar{T}, T),\end{cases}
$$

with $\bar{\mu}>0$ a given parameter. Now we define $r:=\frac{\rho V_{1}}{Q_{1}^{\max }}, \beta:=\frac{V_{1}}{\alpha V_{2}}, \gamma:=\frac{\alpha \kappa}{k}$, and system (1.1) becomes:

$$
\left\{\begin{array}{l}
\frac{d y}{d t}=\frac{\mu(t) y}{1+y}-r y-u y, \\
\frac{d s}{d t}=u \beta(\gamma y-s)-\mu_{2}(s) x, \\
\frac{d x}{d t}=\left(\mu_{2}(s)-u \beta\right) x .
\end{array}\right.
$$

The coupling of the first reactor containing microalgae and the anaerobic digester is therefore modeled by a non-autonomous, three-dimensional controlled system that is linear w.r.t. the input flow $u$. In the case where $\mu$ is given by (1.2), the system is autonomous on each of the two phases (day and night). Next, we assume that $\mu_{2}$ is of type Monod (see [5]), that is

$$
\mu_{2}(s):=\mu_{2}^{m} \frac{s}{K_{s}+s},
$$

where $K_{s}>0$ is the half-saturation coefficient and we denote by $\mu_{2}^{\prime}(s)$ the derivative of $\mu_{2}$ w.r.t. $s$. We also assume that for all $s$, we have

$$
\mu_{2}(s)<\beta .
$$

This assumption is standard in order to control the variation of the biomass concentration in the anaerobic digester, see e.g. [15]. The values of all parameters for system (1.3) are

$$
\bar{\mu}=0.5, r=0.005, T=10, \mu_{2}^{m}=0.1, K_{s}=0.05, \beta=1, \gamma=1 \text {. }
$$

Combining Cauchy-Lipschitz Theorem and Gronwall's Lemma, we can easily see that the set $E:=\mathbb{R}_{+}^{*} \times \mathbb{R}_{+}^{*} \times$ $\mathbb{R}_{+}^{*}$ is an invariant domain for (1.3). In the rest of the paper, we consider only initial conditions in $E$.

\subsection{Optimization problem for methane production}

The objective of this study is to maximize the production of biogas in the second reactor, which is proportional to the production of biomass $\mu_{2}(s(t)) x(t)$. Recall that the control $u$ has been renormalized by the transformation of the system. Over a time interval $[0, n T]$, where $n \in \mathbb{N}^{*}$ is fixed $(n T$ represents the timeframe i.e. a certain number of days), we consider the set of admissible controls

$$
\mathcal{U}_{n}:=\{u:[0, n T] \rightarrow[0,1] \mid \text { meas. }\} .
$$


The optimization problem can be then stated as follows:

$$
\max _{u \in \mathcal{U}_{n}} J_{n}(u):=\int_{0}^{n T} \mu_{2}\left(s_{u}(t)\right) x_{u}(t) d t
$$

where $\left(y_{u}, s_{u}, x_{u}\right)$ is the unique solution of (1.3) starting at a given point $\left(y_{0}, s_{0}, x_{0}\right) \in E$ for a given control $u \in \mathcal{U}_{n}$. To do so, we will focus now on the maximization of $J_{1}$ under periodic constraints, without imposing initial conditions. Thus, we consider the set of periodic controls:

$$
\mathcal{U}:=\{u: \mathbb{R} \rightarrow[0,1] \text { s.t. } u(\cdot) \text { meas., } T-\text { periodic }\} .
$$

We say that a solution $\left(y_{u}, s_{u}, x_{u}\right)$ of $(1.3)$ with a given control $u \in \mathcal{U}$ is $T$-periodic if and only if $\left(y_{u}, s_{u}, x_{u}\right)$ satisfies:

$$
y_{u}(0)=y_{u}(T), \quad s_{u}(0)=s_{u}(T), \quad x_{u}(0)=x_{u}(T) .
$$

Given this constraint, the objective to be maximized becomes

$$
\max _{u \in \mathcal{U}} J_{1}(u)=\int_{0}^{T} \mu_{2}\left(s_{u}(t)\right) x_{u}(t) d t \text {, s.t. (1.5) holds. }
$$

Remark 1.1. From an environmental or economic point of view, the cost function to maximize is $\tilde{J}(u):=$ $\frac{1}{\beta+c} J(u)$ where $c$ is a (environmental or economic) cost ratio of the two processes. Thus, $\beta:=\frac{V_{1}}{\alpha V_{2}}$ is a plant design parameter which should be chosen adequately in order to optimize the coupled process.

Integrating (1.3) over a period, and taking into account the periodicity of the solution yields the equality:

$$
J_{1}(u)=\beta \int_{0}^{T} x_{u}(t) u(t) d t
$$

This expression is used to derive optimality conditions by Pontryagin's Maximum Principle. Before this we will investigate the existence of periodic solutions for system (1.3).

\section{Existence of periodic solutions}

In this section, we are interested in sufficient conditions on a control $u \in \mathcal{U}$ that guarantee the existence of $T$-periodic solutions of (1.3). We first give a general set of assumptions that ensure existence of periodic trajectories of the system (Theorem 2.2), and we study their attraction (Theorem 2.3). We then discuss the case where one hypothesis of Theorem 2.2 is not necessarily satisfied (Theorem 2.4).

\subsection{Sufficient conditions for periodic solutions}

The proof uses the theory of periodic Kolmogorov equations [12, 16, 17, 13]. As we deal with a controlled system, the control $u$ is not fixed, and so is the time-dependency of the dynamics of the system. For the sake of completeness, we have detailed the proof of existence of $T$-periodic solutions for (1.3) in this setting.

Definition 2.1. Given a function $f: \mathbb{R}^{n+1} \rightarrow \mathbb{R}^{n},(t, x) \longmapsto f(t, x)$ which is T-periodic w.r.t. the variable $t$ and bounded in a neighborhood of every point $(t, 0)$, we say that the differential equation

$$
\dot{x}_{i}=x_{i} f_{i}(t, x), 1 \leq i \leq n,(t, x) \in \mathbb{R} \times \mathbb{R}^{n},
$$

is of the Kolmogorov type. Whenever $f$ depends on an additional control variable u, we say that (2.1) is a controlled Kolmogorov equation.

Such systems are useful in biological modeling as the space $\left(\mathbb{R}_{+}^{*}\right)^{n}$ is invariant by $(2.1)$. To prove the existence of periodic solutions of (1.3), we proceed as follows. First, we show that under certain integral assumptions, there exists a unique positive $T$-periodic solution for the concentration of microalgae $y$. Next, we show that $x+s$, which represents the total mass in the second reactor, admits a unique $T$-periodic solution. Finally, we prove the existence of a unique positive periodic solution for the biomass $x$ by a reasoning similar to $y$. 
Let us define the function $F: \mathbb{R} \times[0,+\infty) \times[0,1] \rightarrow \mathbb{R}$ by:

$$
F(t, y, u):=\frac{\mu(t)}{1+y}-(r+u),
$$

hence the micro-algae concentration can be put into a one-dimensional controlled Kolmogorov equation:

$$
\dot{y}=y F(t, y, u(t)) .
$$

We recall the following Theorem, a variation of a result in [13] that is proved in [18]. It provides a general existence result for a one-dimensional controlled Kolmogorov equation.

Theorem 2.1. Let $G: \mathbb{R} \times[0,+\infty) \times[0,1] \rightarrow \mathbb{R}$ be a function measurable w.r.t. the pair $(t, u)$, local-Lipschitz continuous w.r.t. $x$, and $T$-periodic w.r.t. to $t$ and non-increasing w.r.t. u. Suppose that there exists $M>0$ and $s_{0}, s_{1} \in[0, T]$ with $s_{0}<s_{1}$ such that:

(i) $\int_{0}^{T} G(t, 0,1) d t>0>\int_{0}^{T} G(t, M, 0) d t$.

(ii) For all $t \in[0, T]$, for all $u \in[0,1], G(t, \cdot, u)$ is non-increasing.

(iii) For all $t \in\left[s_{0}, s_{1}\right]$, for all $u \in[0,1], G(t, \cdot, u)$ is decreasing.

Then, the equation

$$
\dot{y}=y G(t, y, u(t)),
$$

admits a unique positive T-periodic solution. Moreover, the set of initial conditions for which there exists a periodic solution of (2.4) coincides with the interval $\left[z_{1}, z_{0}\right]$ where $z_{1}$ (resp. $\left.z_{0}\right)$ is the unique initial condition of the T-periodic positive solution of (2.4) with a constant control $u=1$ (resp. $u=0$ ).

Next, we consider the following condition on the system:

Assumption 2.1. We suppose that:

$$
\int_{0}^{T} \mu(t) d t>(r+1) T .
$$

Assumption 2.1 means that one cannot operate the digester at a too high dilution rate which prevents wash-out. Notice that (2.5) implies that:

$$
\int_{0}^{T} \mu(t) d t>r T .
$$

Inequality (2.6) means that microalgae can survive in batch mode (without dilution) and that there exists a periodic solution of (2.3) for the control $u=0$ (see Proposition 2.1). Whenever the concentration of microalgae is low, growth during the day will compensate the loss of micro-algae by respiration during the night.

Throughout this section, we will suppose that (2.5) holds whereas in section 2.3 , we only suppose $(2.6)$ (which is less restrictive). The periodicity of $y$ is a direct consequence of Theorem 2.1.

Proposition 2.1. Assume that the parameters of the system satisfy (2.5). Then:

(i) For all $u \in \mathcal{U}$ there exists a unique T-periodic positive solution of (2.3) denoted $\tilde{y}_{u}$.

(ii) The set of initial conditions for which (2.3) is periodic is the interval $\left[z_{0}, z_{1}\right]$, where $z_{1}$ (resp. $z_{0}$ ) is the initial condition of the T-periodic positive solution $y_{1}$ (resp. $\left.y_{0}\right)$ with a constant control $u=1$ (resp. $u=0$ ).

Proof. It is straightforward to check that (2.5) implies the inequality: $\int_{0}^{T} F(t, 0,1) d t>0$. Moreover, one has

$$
\int_{0}^{T} F(t, M, 0) d t=\frac{1}{1+M} \int_{0}^{T} \mu(t) d t-r T
$$

which is negative if $M$ is chosen large enough. Assumptions (ii) and (iii) of Theorem 2.1 are easy to verify. We are now in position to apply Theorem 2.1 which gives the result.

The second step consists in proving the existence of a periodic solution for $\psi:=x+s$ which satisfies the linear equation:

$$
\dot{\psi}=\beta u\left(\gamma \tilde{y}_{u}-\psi\right) .
$$

We denote by $\|u\|_{1}$ the $L^{1}$-norm of the control on the interval $[0, T]$, that is, $\|u\|_{1}:=\int_{0}^{T} u(t) d t$. We then have the following result. 
Proposition 2.2. For all $u \in \mathcal{U}$, equation (2.7) admits a unique positive T-periodic solution $\tilde{\psi}_{u}$.

Proof. Whenever $u \equiv 0, \psi$ is constant (equal to $x_{0}+s_{0}>0$ ) and $T$-periodic. We may now assume that $\|u\|_{1} \neq 0$. From the linearity of (2.7), one can check immediately that

$$
\tilde{\psi}_{u}(t):=\beta \gamma \int_{0}^{t} u(s) \tilde{y}_{u}(s) e^{\int_{t}^{s} \beta u(\sigma) d \sigma} d s+\beta \gamma \frac{\int_{0}^{T} u(s) \tilde{y}_{u}(s) e^{\int_{T}^{s} \beta u(\sigma) d \sigma} d s}{1-e^{-\int_{0}^{T} \beta u(s) d s}} e^{-\int_{0}^{t} \beta u(s) d s},
$$

is well defined (as $\|u\|_{1} \neq 0$ ) and is the unique periodic solution of (2.7).

It remains to show the existence of an initial condition for which $x$ is periodic. Given $u \in \mathcal{U}$, we can rewrite the equation satisfied by $x$ as follows:

$$
\dot{x}=x\left[\mu_{2}\left(\tilde{\psi}_{u}(t)-x\right)-\beta u(t)\right]
$$

and we can use the same scheme as for proving the existence of a periodic solution for $y$. Notice that if $u \equiv 0$, then a solution of (2.9) is strictly increasing, so there is no periodic solution of (1.3) for this control. We can now assume that $\|u\|_{1} \neq 0$.

Proposition 2.3. Assume that $u \in \mathcal{U}$ is such that $\|u\|_{1} \neq 0$ and

$$
\|u\|_{1}<\frac{1}{\beta} \int_{0}^{T} \mu_{2}\left(\tilde{\psi}_{u}(t)\right) d t
$$

Then, there exists a T-periodic solution $\tilde{x}_{u}$ of $(2.9)$.

Proof. Let us define a function $\tilde{G}: \mathbb{R} \times[0,+\infty) \times[0,1] \rightarrow \mathbb{R}$ be defined by $\tilde{G}(t, x):=\mu_{2}\left(\tilde{\psi}_{u}(t)-x\right)-\beta u(t)$. Equation (2.9) is a Kolmogorov equation, and it can be written:

$$
\dot{x}=x \tilde{G}(t, x),
$$

Using (2.10), we can apply Theorem 2.3 of [18] which shows the existence of a periodic solution for (2.9).

Now, if we set $\tilde{s}_{u}:=\tilde{\psi}_{u}-\tilde{x}_{u}$, we have $\dot{\tilde{s}}_{u}=u \beta\left(\gamma \tilde{y}_{u}-\tilde{s}_{u}\right)-\mu_{2}\left(\tilde{s}_{u}\right) \tilde{x}_{u}$. Moreover, as initial conditions are in $E$ which is invariant by $(1.3)$, we have $\tilde{s}_{u}(t) \geq 0$ for all $t$. Hence, we have obtained the existence of a positive periodic solution to (1.3) provided that the additional condition (2.10) is satisfied.

Inequality $(2.10)$ is intricate as $\tilde{\psi}_{u}$ depends on the control $u$. The next lemma provides a sufficient condition ensuring the existence of controls $u$ which satisfy (2.10). It will lead to Theorem 2.2 which is the main result of this part.

Lemma 2.1. If Assumption 2.1 is satisfied, any positive T-periodic solution of (2.3) satisfies for all $t \in[0, T]$ :

$$
y(t) \geq y_{1}(t) .
$$

Proof. The inequality is a consequence of the monotonicity property of $u \longmapsto F(t, x, u)$ and Proposition 2.1.

Next, denote by $z_{1}^{-}$the minimum of $y_{1}$ over $[0, T]$. Gathering Propositions 2.1, 2.2, 2.3 and Lemma 2.1 yields the following result.

Theorem 2.2. Suppose that Assumption 2.1 is satisfied. Then, for all $u \in \mathcal{U}$ with $\|u\|_{1} \neq 0$ such that

$$
\|u\|_{1}<\frac{T}{\beta} \mu_{2}\left(\gamma z_{1}^{-}\right)
$$

there exists a T-periodic solution $\left(\tilde{y}_{u}(\cdot), \tilde{s}_{u}(\cdot), \tilde{x}_{u}(\cdot)\right)$ of $(1.3)$.

Proof. Combining (2.8) and (2.11) yields that $\tilde{\psi}_{u}(t) \geq \gamma z_{1}^{-}$for all $t \in[0, T]$ (notice that if $\tilde{y}_{u}$ is constant in (2.8), then $\left.\tilde{\psi}_{u}=\gamma \tilde{y}_{u}\right)$. Therefore, (2.12) implies that $\|u\|_{1}<\frac{1}{\beta} \int_{0}^{T} \mu_{2}\left(\tilde{\psi}_{u}(t)\right) d t$, and thus (2.10) is satisfied. We can now apply Propositions 2.1 and 2.3 to get the existence of periodic solutions $\tilde{y}_{u}$ and $\tilde{\psi}_{u}$ of $(2.3)$-(2.7). Moreover, as (2.10) is satisfied, Proposition 2.1 implies the existence of a $T$-periodic solution $\tilde{x}_{u}$ of $(2.9)$. Now, $\tilde{s}_{u}:=\tilde{\psi}_{u}-\tilde{x}_{u}$ is also $T$-periodic and positive which concludes the proof. 
Notice that initial conditions for which the system is periodic are part of the unknown for problem (1.6). Hence, Proposition 2.1 provides an exact description of the set of initial conditions for which the solution of (2.3) is periodic. This property is useful from a numerical point of view for choosing a maximal and a minimal bound for initial conditions. Characterizing in a similar way the set of initial conditions for which $x(\cdot)$ is periodic is a more difficult question as $\tilde{\psi}_{u}$ depends on the control $u$. One can prove the following property concerning the initial value of $\tilde{\psi}_{u}$.

Lemma 2.2. Let $z_{0}^{+}$the maximal value of $y_{0}$. Then, for any $u \in \mathcal{U}$, we have $\tilde{\psi}_{u}(0) \in\left[z_{1}^{-}, z_{0}^{+}\right]$.

Proof. If $\tilde{y}_{u}$ is constant in (2.8), then we have $\tilde{\psi}_{u}=\gamma \tilde{y}_{u}$ (by uniqueness of the periodic solution of (2.7)). This proves the result.

\subsection{Attraction of periodic solutions}

In this section, we prove that given a periodic control $u \in \mathcal{U}$, the unique periodic solution $\left(\tilde{y}_{u}, \tilde{s}_{u}, \tilde{x}_{u}\right)$ of $(1.3)$ provided by Theorem 2.2 is attractive in the following sense.

Theorem 2.3. Suppose that Assumption 2.1 together with (2.12) hold. Given a non-zero control $u \in \mathcal{U}$ and a triple $\zeta_{0}:=\left(y_{0}, s_{0}, x_{0}\right) \in \mathbb{R}_{+}^{*}$, let $(y, s, x)$ the unique solution of $(1.3)$ starting at $\zeta_{0}$. Then, we have:

$$
\lim _{t \rightarrow+\infty}\left|y(t)-\tilde{y}_{u}(t)\right|=\lim _{t \rightarrow+\infty}\left|s(t)-\tilde{s}_{u}(t)\right|=\lim _{t \rightarrow+\infty}\left|x(t)-\tilde{x}_{u}(t)\right|=0,
$$

where $\left(\tilde{y}_{u}, \tilde{s}_{u}, \tilde{x}_{u}\right)$ is the unique T-periodic solution of (1.3) associated to control $u$.

Proof. To prove that $\lim _{t \rightarrow+\infty}\left|y(t)-\tilde{y}_{u}(t)\right|=0$, we can use the classical property of attraction of Kolmogorov periodic equations (see [13] or Theorem 2.4 in [18]). Let us now show that $x+s$ converges to $\tilde{x}_{u}+\tilde{s}_{u}$. To this end, let $\psi:=x+s, z:=y-\tilde{y}_{u}, \varepsilon>0$ and fix $t_{0}>0$ such that $|z(t)| \leq \varepsilon$ for all $t \geq t_{0}$. By using (2.8), we have:

$$
\psi(t)-\tilde{\psi}_{u}(t)=\beta \gamma \int_{0}^{t} z(s) u(s) d s e^{\int_{0}^{s} \beta u(\sigma) d \sigma} e^{-\int_{0}^{t} \beta u(s) d s}+\left[\psi(0)-\tilde{\psi}_{u}(0)\right] e^{-\int_{0}^{t} \beta u(s) d s} .
$$

It is enough to show that the first term in (2.13) goes to zero. We have for $t \geq t_{0}$

$$
\begin{aligned}
\mid \int_{0}^{t} z(s) u(s) e^{\int_{0}^{s} \beta u(\sigma) d \sigma} d s e^{-\int_{0}^{t} \beta u(s) d s \mid} & \leq \int_{0}^{t_{0}}|z(s)| u(s) e^{\int_{0}^{s} \beta u(\sigma) d \sigma} d s e^{-\int_{0}^{t} \beta u(s) d s} \\
& +\int_{t_{0}}^{t}|z(s)| u(s) e^{\int_{0}^{s} \beta u(\sigma) d \sigma} d s e^{-\int_{0}^{t} \beta u(s) d s} \\
\leq & \int_{0}^{t_{0}}|z(s)| u(s) e^{\int_{0}^{s} \beta u(\sigma) d \sigma} d s e^{-\int_{0}^{t} \beta u(s) d s}+\varepsilon\left[1-e^{-\int_{t_{0}}^{t} \beta u(s) d s}\right]
\end{aligned}
$$

The second term in the right member of the previous inequality is bounded by $\varepsilon$ and the first term goes to zero when $t$ goes to infinity. Hence, we obtain that $\psi(t)-\tilde{\psi}_{u}(t)$ goes to zero when $t$ goes to infinity.

It remains to prove that $x(t)-\tilde{x}_{u}(t)$ goes to zero when $t$ goes to infinity. To this end, let us define $\bar{x}$ as the unique solution of the Cauchy problem

$$
\dot{w}=\left[\mu_{2}\left(\tilde{\psi}_{u}(t)-w\right)-\beta u(t)\right] w, w(0)=x_{0} .
$$

As previously, we can use Theorem 2.4 in [18] or [13] to show that $\bar{x}(t)-\tilde{x}_{u}(t)$ goes to zero when $t$ goes to infinity.

Lemma 2.3. The function $\bar{x}(t)-x(t)$ converges to zero when $t$ goes to infinity.

This lemma is rather technical and is proved in the appendix. This concludes the proof of the theorem. 


\subsection{Discussion of Assumption 2.1}

In this part, we prove that there exist $T$-periodic solutions of (1.3) whenever (2.5) is replaced by (2.6). In fact, (2.5) can be restrictive from a practical point of view. This will be possible by considering an upper bound in $L^{\infty}$ for admissible controls (and thus a smaller class of admissible controls).

Let us set $\delta:=\int_{0}^{T} \mu(t) d t-r T$. So, (2.6) implies that $\delta>0$ and that there exists $\varepsilon>0$ such that

$$
\int_{0}^{T} \mu(t) d t>(r+\varepsilon) T
$$

We also suppose that Assumption 2.1 does not hold, i.e. $\delta<T$.

Proposition 2.4. (i) If $u \in \mathcal{U}$ is such that $\|u\|_{1}<\delta$, there exists a unique positive periodic solution of (2.3). (ii) The set of initial conditions for which (2.3) is T-periodic is $\left(0, z_{0}\right]$.

Proof. Let us given a control $u \in \mathcal{U}$. A direct computation gives:

$$
\int_{0}^{T} G(t, 0) d t=\delta-\int_{0}^{T} u(t) d t>0, \int_{0}^{T} G(t, M) d t=\frac{1}{1+M} \int_{0}^{T} \mu(t) d t-r T-\int_{0}^{T} u(t) d t,
$$

where we have set $G(t, y):=F(t, y, u(t))$ (recall (2.2)). Moreover, by taking $M$ large enough, it is possible to assume that the second integral in (2.15) is negative. We can apply Theorem 2.3 in [18] to conclude for (i). To prove (ii), consider the mapping $y_{0} \longmapsto y_{1}(T)$ where $y_{1}$ is the unique solution of $(2.3)$ starting at $y_{0}$ at time 0 with the constant control $u=1$. By dividing (2.3) by $y$ and integrating this equation over $[0, T]$, we obtain using $\delta<T$ that this mapping is decreasing on $\left(0, z_{0}\right]$. Moreover, as $z_{0}$ is the unique initial condition for the control $u=0$, the mapping $y_{0} \longmapsto y_{0}(T)$ is increasing (where $y_{0}$ is the unique solution of (2.3) starting at $y_{0}$ at time 0). We obtain (ii) by a similar argument as for proving Theorem 2.1 in [18].

Remark 2.1. (i) When (2.5) is no longer satisfied, it is delicate to obtain a lower bound on $\tilde{\psi}_{u}$ in (2.10) implying an inequality such as (2.12). In fact, the infimum of the set of initial conditions for which (2.3) is periodic is zero, see Proposition 2.4 (ii).

(ii) To overcome this difficulty, we will consider controls taking values within $[0, \varepsilon]$, and we will repeat the arguments of section 2.1 by replacing the maximal control value by $\varepsilon$.

Next, we consider the set $\mathcal{U}_{\varepsilon}$ which consists of admissible controls that are $T$-periodic and that take values within $[0, \varepsilon]$. Following Proposition 2.1, one can show that there exists a unique solution $y_{\varepsilon}$ of $(2.3)$ for the control $u=\varepsilon$, and we denote by $z_{\varepsilon}^{-}>0$ its minimal value.

Theorem 2.4. For all $u \in \mathcal{U}_{\varepsilon}$ with $\|u\|_{1} \neq 0$ such that

$$
\|u\|_{1}<\frac{T}{\beta} \mu_{2}\left(\gamma z_{\varepsilon}^{-}\right)
$$

there exists a T-periodic solution $\left(\tilde{y}_{u}(\cdot), \tilde{s}_{u}(\cdot), \tilde{x}_{u}(\cdot)\right)$ of $(1.3)$.

Proof. The existence of a $T$-periodic solution of (2.7) is similar to the proof of Proposition 2.2. To prove the existence of a periodic solution of (2.9), we proceed as in the proof of Proposition 2.3. Now, inequality (2.16) implies (2.10) as in Theorem 2.2. This proves the Theorem.

\subsection{Existence of an optimal control}

The existence of an optimal control for problem (1.6) is rather standard except in the case where $\mu=\mu_{0}$. Indeed, the dynamics is no longer continuous at $t=\bar{T}$, but as the discontinuity point of the dynamics is fixed, the existence of an optimal control can be easily proved by the following proposition.

Proposition 2.5. Assume that $\mu=\mu_{0}$ and that there exists a control $u \in \mathcal{U}$ such that the solution of (1.3) is T-periodic. Then, there exists an optimal control for problem (1.6). 
Proof. First, admissible controls take values within a compact interval, and system (1.3) is affine w.r.t. the control. Let us given $u \in \mathcal{U}$ and let $\left(y_{u}, s_{u}, x_{u}\right)$ its associated solution. In view of the discontinuity point of $\mu$ at $t=\bar{T}$, we set:

$$
y_{1}(t):=y_{u}(t), t \in[0, \bar{T}], y_{2}(t):=y_{u}\left(\frac{T-\bar{T}}{\bar{T}} t+\bar{T}\right), t \in[0, \bar{T}] .
$$

Similarly, we can define $\left(s_{1}, s_{2}\right)$ and $\left(x_{1}, x_{2}\right)$. Hence, system (1.3) can be embedded into a six-dimensional system $\left(y_{1}, y_{2}, s_{1}, s_{2}, x_{1}, x_{2}\right)$ with one phase together with additional continuity assumptions $y_{2}(0)=y_{1}(\bar{T})$, $s_{2}(0)=s_{1}(\bar{T})$ and $x_{2}(0)=x_{1}(\bar{T})$. Similarly, the cost function can be put changed into an integral over $[0, \bar{T}]$. As a consequence, we can apply Fillipov's existence Theorem [19] with a continuous dynamics and cost function, the set of periodic trajectories being non-empty. This concludes the proof.

\section{Necessary conditions on optimal trajectories}

In this section, we apply the Pontryagin Maximum Principle (see [20]) to derive necessary conditions on optimal trajectories for problem (1.6) that will be used to implement an indirect shooting method.

\subsection{Pontryagin maximum principle}

Let $z:=(y, x, u)$ and $\lambda:=\left(\lambda_{1}, \lambda_{2}, \lambda_{3}\right)$ the adjoint vector. First, we define the Hamiltonian $H:=H\left(z, \lambda, \lambda_{0}, u\right)$ associated to (1.6) by:

$$
H:=\lambda_{1}\left[\mu \frac{y}{1+y}-r y\right]+\left(\lambda_{3}-\lambda_{2}\right) \mu_{2}(s) x+u\left(-\lambda_{1} y+\lambda_{2} \beta(\gamma y-s)-\lambda_{3} \beta x+\lambda_{0} x\right) .
$$

Let $u$ an optimal control and $z_{u}:=\left(y_{u}(\cdot), x_{u}(\cdot), s_{u}(\cdot)\right)$ the associated $T$-periodic trajectory. Then, there exists $\lambda_{0} \leq 0$ and an absolutely continuous map $\lambda:[0, T] \rightarrow \mathbb{R}^{3}$ such that the adjoint equations $\dot{\lambda}=$ $-\frac{\partial H}{\partial x}\left(z_{u}(t), \lambda(t), \lambda_{0}, u(t)\right)$ are satisfied, that is:

$$
\left\{\begin{array}{l}
\frac{d \lambda_{1}}{d t}=\left(-\frac{\mu}{(1+y)^{2}}+r+u\right) \lambda_{1}-u \lambda_{2} \beta \gamma \\
\frac{d \lambda_{2}}{d t}=x\left(\lambda_{2}-\lambda_{3}\right) \mu_{2}^{\prime}(s)+u \lambda_{2} \beta \\
\frac{d \lambda_{3}}{d t}=\left(\lambda_{2}-\lambda_{3}\right) \mu_{2}(s)+u \lambda_{3} \beta+\lambda_{0} u
\end{array}\right.
$$

As the state is $T$-periodic, the adjoint vector $\lambda$ satisfies the following transversality condition:

$$
\lambda(0)=\lambda(T)
$$

i.e. $t \longmapsto \lambda(t)$ is also $T$-periodic. Finally, we have the maximization condition: for a.e. $t \in[0, T], u(t)$ maximizes the function

$$
v \longmapsto H\left(t, z(t), \lambda(t), \lambda_{0}, v\right) .
$$

If the real $\lambda_{0}$ can take the value zero, the trajectory is said abnormal. It corresponds to a degenerate case of the optimization problem where optimal controls are independent of the cost function. Next, we assume that the optimal trajectory is normal, that is $\lambda_{0} \neq 0$ (by homogeneity, we take $\lambda_{0}=-1$ ). The switching function associated to the control is

$$
\phi:=-\lambda_{1} y+\lambda_{2} \beta(\gamma y-s)-\lambda_{3} \beta x+x,
$$

and it is $T$-periodic. From (3.1) an optimal control satisfies the following control law:

$$
\left\{\begin{array}{l}
u=1 \Longleftrightarrow \phi>0 \\
u=0 \Longleftrightarrow \phi<0
\end{array}\right.
$$

When $u=1$ (resp. $u=0$ ) on some time interval, we say that the trajectory has an arc Bang $g_{+}$(resp. Bang_). We also denote by $B_{ \pm}$an arc Bang + or Bang_. If $\phi$ is zero on some time interval, we say that the trajectory has a singular arc which means that the corresponding singular control takes value in $(0,1)$. We call switching point a time $t_{0}$ at which the control is non-constant in any neighborhood of $t_{0}$. In particular the switching function satisfies $\phi\left(t_{0}\right)=0$. 
From (1.3) and (1.4), we can see that the constant controls $u=0$ and $u=1$ are not solution of the problem (otherwise $x$ would be either increasing or decreasing on $[0, T]$ in contradiction with the periodicity). It follows that the optimal solution $\left(y_{u}, s_{u}, x_{u}\right)$ either contains a switching point or it is singular on $[0, T]$. The periodicity of the switching function implies also the following property.

- If $\phi(0)>0$, then the control satisfies $u=0$ on $\left[0, t_{0}\right] \cup\left[t_{1}, T\right]$ where $0<t_{0}<t_{1}<T$. Moreover, the trajectory contains at least two switching points on $[0, T]$.

- If $\phi(0)<0$, then the control satisfies $u=1$ on $\left[0, t_{0}^{\prime}\right] \cup\left[t_{1}^{\prime}, T\right]$ where $0<t_{0}^{\prime}<t_{1}^{\prime}<T$. Moreover, the trajectory contains at least two switching points on $[0, T]$.

In other words, the periodic assumptions on the state-adjoint system imply that the trajectory has the same structure in a neigbourhood of $t=0$ and of $t=T$ whenever $\phi(0)>0$ or $\phi(0)<0$.

\subsection{Computation of the singular arcs}

In this section, we explain how singular arcs can be computed. The exact computations are given in the appendix. Let $\left[t_{1}, t_{2}\right]$ be a time interval where the optimal trajectory contains a singular arc. We thus have:

$$
\phi(t)=0, \forall t \in\left[t_{1}, t_{2}\right]
$$

In order to compute the singular control in terms of the state-adjoint vector, we differentiate $\phi$ (which is absolutely continuous) w.r.t. $t$. Thus, we obtain for all $t \in\left[t_{1}, t_{2}\right]$ :

$$
\dot{\phi}(t)=(\gamma y-s)\left(\lambda_{2}-\lambda_{3}\right) \beta x \mu_{2}^{\prime}(s)+\mu_{2}(s) x+y\left[-\lambda_{1} y \frac{\mu(t)}{(1+y)^{2}}+\lambda_{2} \beta \gamma \frac{\mu(t)}{1+y}-r \beta \gamma \lambda_{2}\right] .
$$

When $\mu(t)$ is given by (1.2), $\phi^{\prime}$ is not necessarily continuous by (3.3) as the system is autonomous on each interval $[0, \bar{T})$ and $(\bar{T}, T]$. Thus, in this case, we differentiate the expression $(3.2)$ on both intervals $[0, \bar{T})$ and $(\bar{T}, T]$. The derivative of $\dot{\phi}$ can be written:

$$
\ddot{\phi}(t)=A_{\mu}(z(t), \lambda(t))+u(t) B_{\mu}(z(t), \lambda(t)),
$$

where $A_{\mu}$ and $B_{\mu}$ depend on $(z, \lambda)$. Exact expressions of $\ddot{\phi}$ for a general function $\mu(\cdot)$ can be found in the appendix.

For numerical verifications of optimality of the singular arc, it is important to recall Legendre-Clebsch condition: if the singular arc is optimal, then we must have:

$$
B_{\mu}(z(t), \lambda(t)) \geq 0
$$

see e.g. [21]. Moreover, if $B_{\mu}(z(t), \lambda(t))>0$, then the singular control $u_{s}$ is given by:

$$
u_{s}(t):=-\frac{A_{\mu}(z(t), \lambda(t))}{B_{\mu}(z(t), \lambda(t))}
$$

When (3.5) defines a control in [0,1], we say that the singular arc is controllable.

\section{Numerical Results}

\subsection{Direct and indirect methods}

The direct approach transforms the infinite dimensional optimal control problem into a finite dimensional optimization problem. This is done by a discretization in time applied to the state and control variables, and dynamics. The resulting problem can be solved by a classical NLP software. We refer the reader to [22] and [23] for more details on direct transcription methods and NLP algorithms.

The indirect approach relies on Pontryagin's Maximum Principle to express the optimal control as a maximizer of a Hamiltonian. This Hamiltonian depends both on the state and the adjoint variables, which are solution of a boundary value problem. This BVP is still in an infinite dimensional setting, and it can be solved 
for instance by shooting methods (see [24, 25])

In our experiments, we first try the direct approach, starting from a very simple initialization with constant values for the control and state variables over time. Then we analyze the obtained solution, and we determine the structure of the optimal control (number and type of arcs). In addition to this qualitative information, we also extract numerical estimates for the switching times, state and costate variables. We recall that the co-state (or adjoint state) for Pontryagin's Principle correspond to the Lagrange multipliers for the dynamics constraints in the discretized problem. This allows to verify numerically Legendre-Clebsch condition (3.4).

Here are the settings for both methods.

Direct. Software Bocop[10], discretization by a Lobatto IIIC formula (6th order) with 100 time steps per day, constant initialization, tolerance for NLP solver set at $10^{-10}$.

Indirect. Software Shoot[11], tolerances set at $10^{-12}$ for the ODE solver and $10^{-14}$ for the quasi-Newton solver, initialization from the direct solution.

Computation times on a Xeon $3.2 \mathrm{GHz}$ are less than one minute for the direct method, and less than one second for the indirect method. In all the following figures, the x-axis represents time with the scale $\mathbf{1 0}$ for 1 day, except when specified otherwise.

\subsection{Impact of the light model on the singular control}

We begin the numerical simulations with the discontinuous light model (1.2) and some regularized variants. We observe that these models, while being convenient for the mathematical analysis of the problem, suffer from their non-smoothness that translates into the singular control. Indeed, for the discontinuous case, the control structure itself is changed, as the singular arc cannot cross the discontinuity (see section 5.3).

\subsubsection{Discontinuous light model}

The preliminary simulations use the discontinuous light model (1.2). We first try to solve the problem with the direct method, from a basic constant initialization. The control structure seems to be $B^{-} S B^{-}$, namely we start with a bang $\operatorname{arc} u=0$, followed by a singular $\operatorname{arc}$ that crosses $\bar{T}$, and finally a second bang $\operatorname{arc} u=0$. We also observe some kind of numerical artifact around $\bar{T}$, where the growth function, and therefore the dynamics of the system, is discontinuous. However, even initialized with the data from the direct solution, the indirect method fails to converge to a solution with this structure.

Upon closer examination, the direct solution suggests a very small bang arc $u=1$ across $\bar{T}$. Setting this time the structure as $B^{-} S B^{+} S B^{-}$, the indirect method converges easily, with the switching times $[0.558778,4.999637,5.000382,9.309402]$. We show on Fig. 2 the control over time, as computed by the direct and indirect methods. First, the singular arc is controllable. Then, we observe that the singular control found by the direct method matches very well the analytical expression used in the indirect method (see Appendix 5.2) and that it satisfies Legendre-Clebsch condition (3.4).

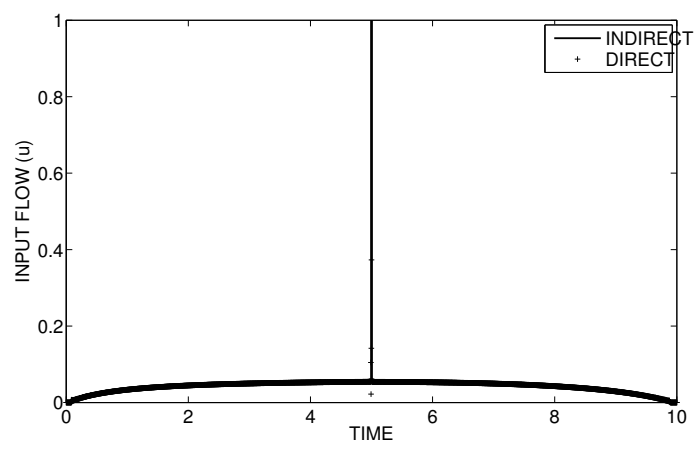

Figure 2: Discontinuous light model - Input-flow $(\mathrm{u})$ : direct and indirect solutions. 
Table 1: Comparison of the direct and indirect methods.

\begin{tabular}{l|r|r|r|r} 
Method & Objective & $\mathrm{y}(0)$ & $\mathrm{s}(0)$ & $\mathrm{x}(0)$ \\
\hline Direct & 4.03635 & 8.46848 & $2.812 \mathrm{E}-04$ & 9.7109 \\
Indirect & 4.036346 & 8.467771 & $2.829097 \mathrm{E}-04$ & 9.710909
\end{tabular}

We also check the control structure and draw the switching function and its first time derivative on Fig. 3. We observe that $\phi$ is negative when $u=0$, as expected from the Maximum Principle. Also, both $\phi$ and $\dot{ }$ are equal to zero over the two singular arcs where the control $u \in(0,1)$. Finally, we can see the discontinuous jump on $\dot{\phi}$ at $\bar{T}$, and the small bang arc that allows to cross this discontinuity while connecting the two singular arcs where $\dot{\phi}=0$. A thorough discussion of this case is presented in Appendix 5.3.
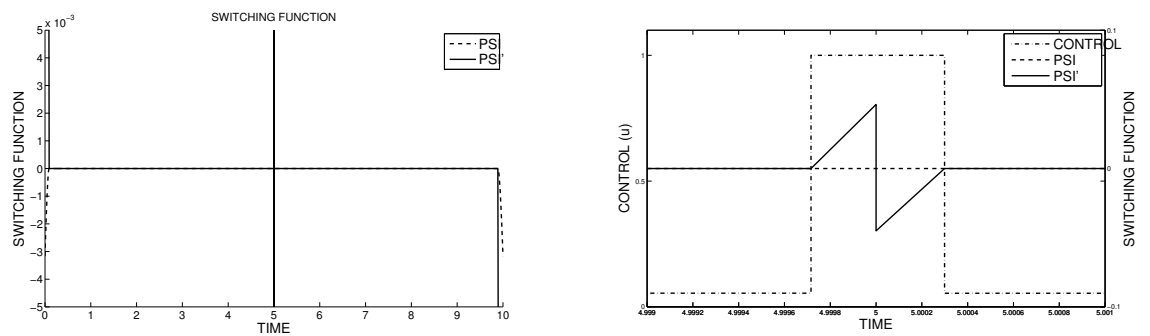

Figure 3: Switching function and its first derivative (zoom on the discontinuity).

\subsubsection{Regularized $C^{0}$ and $C^{1}$ light models}

We now try two regularized versions of (1.2), continuous and $C^{1}$, shown on Fig. 4. The continuous model has a linear junction between day and night, and the $C^{1}$ model has two quadratic junctions. The smoothness of $\mu$ impacts the smoothness of the switching function $\phi$, and therefore the smoothness of the singular control. We saw in 3.2 that the optimal control over a singular arc is the value that equates $\ddot{\phi}$ to 0 . As $\dot{\phi}$ has here the same smoothness as $\mu$, the three light models give the situations:

a) Discontinuous light. If $\mu$ is discontinuous, there is a jump in $\dot{\phi}$. As we must have $\phi=\dot{\phi}=0$ over a singular arc, we see that a singular arc cannot cross the discontinuity in the general case, see Appendix 5.3 for more details. Numerically, we indeed observed a very small bang arc at the discontinuity of $\mu$.

b) Continuous light. If $\mu$ is continuous but not $C^{1}$, a singular arc can cross the points where $\mu$ is not $C^{1}$, as $\dot{\phi}$ stays equal to 0 . However, $\ddot{\phi}$ will be discontinuous, therefore we expect the singular control to be also discontinuous.

c) $C^{1}$ light model. If $\mu$ is $C^{1}, \ddot{\phi}$ is continuous, and we expect the singular control to be continuous as well. It will not be $C^{1}$, however, at the points where $\mu$ is not $C^{2}$.

The three case are illustrated on Fig. 4. Note that apart from this qualitative differences due to the smoothness of the light model, the three solutions are almost identical with respect to the state and control variables obtained after optimization.

\subsection{Optimization with an oscillating light model (smooth)}

In all the following of this section, the simulations use a more realistic, smooth $\left(C^{2}\right)$, periodic light model:

$$
\mu(t)=\bar{\mu} \max \left(0, \sin \left(\frac{2 \pi t}{T}\right)\right)^{2}, \quad \forall t \in[0, T] .
$$

\subsubsection{Optimal 1-day periodic strategy}

We start the simulations with the oscillating light model (4.1), by optimizing the methane production over 1 day (ie $t_{f}=T=10$ ), with periodicity conditions on the state variables $(y, s, x)$. We set the bounds $y(\cdot) \in[0.1,100], s(\cdot) \in[0,100], x(\cdot) \in[0,100]$, and use the constant starting point $y(\cdot)=s(\cdot)=x(\cdot)=50$, with 

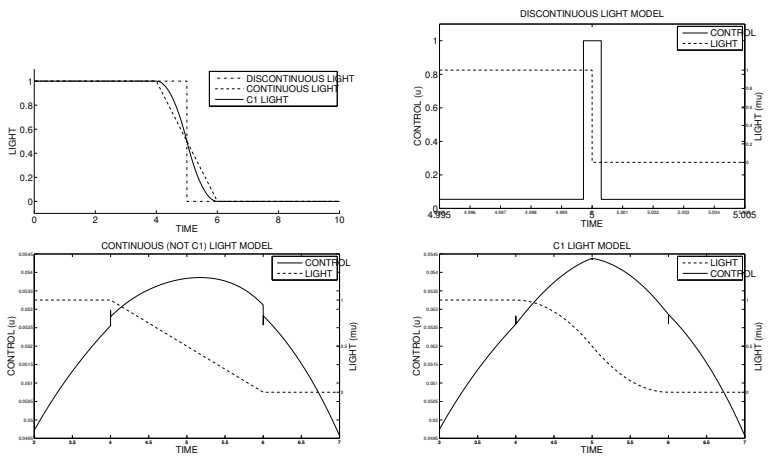

Figure 4: Three light models and corresponding singular control.

$u(\cdot)=0.1$

We obtain the solution shown on Fig. 5, with the initial (and here final) conditions $\zeta_{0}^{*}=(5.66,1.7 E-$ $6,6.46)$. The control structure is $B^{-} S B^{-}$with the singular arc over the time interval [1.69, 9.45]. During the first bang arc, the flow is null and both reactors are in batch mode. We observe that microalgae begin to grow (recall that we are during the day) while biomass in the second reactor stays constant, as there is no substrate available. This corresponds to a null production, indicating that the second reactor is probably oversized. Then the singular arc begins and microalgae pour into the second reactor. Microalgae still grow in the first reactor for a while, then begin to decrease a bit before $\bar{T}$ (the nightfall). Meanwhile in the second reactor, the biomass decreases and the concentration of substrate rises, until substrate is high enough so that biomass begins to grow. Substrate then decreases in turn until it reaches 0 again, while the biomass stabilizes around its intial value. The cycle ends on a small bang arc where the reactors are back in batch mode. Note that the relative variations of $(y, s, x)$ are rather small, which may be due to the rather slow dynamics of the bioprocesses.

We obtain that the control satisfies $u=0$ near $t=0$ and $t=\bar{T}$ which is confirmed by the Pontryagin Maximum Principle. In fact, as the switching function is periodic, the control should be the same near $t=0$ and $t=\bar{T}$.
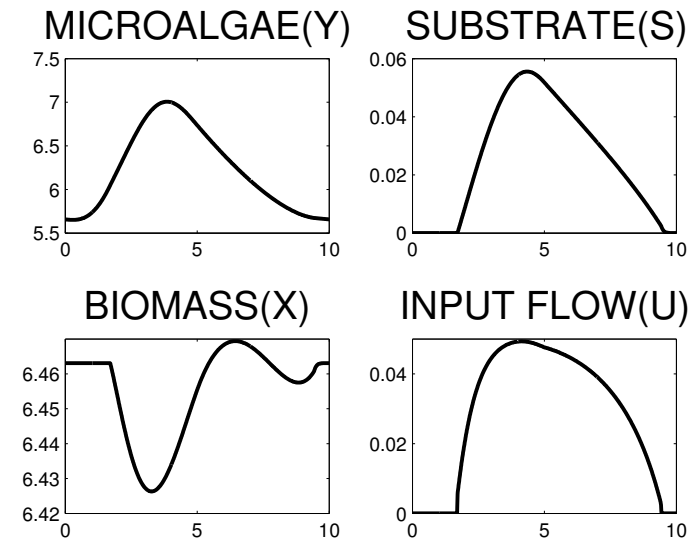

Figure 5: Optimization of a 1-day periodic cycle.

Let us now illustrate the attraction result from Theorem 2.3. We take the control $u$ obtained from the optimization of one periodic day-night cycle, as shown on Fig. 5. We now apply this same control repeatedly for 30 days on the system, starting with the same initial conditions found during the previous initialization, namely $\zeta_{0}^{*}$. The periodic trajectory we obtain is drawn in solid line on Fig. 6 (microalgae and biomass concentrations over time). Now we apply the same control, but starting from different initial conditions, 
$(20,10,2)$ and $(1,5,1)$. We observe that the corresponding trajectories, while not periodic, indeed converge to the periodic one as time grows.
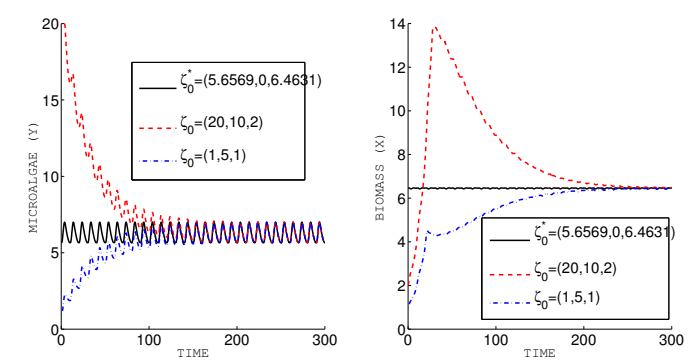

Figure 6: Attraction of the periodic trajectories - Microalgae and biomass over time.

\subsubsection{Optimization over $\mathrm{N}$ days}

Here we study the optimal trajectories for longer timeframes, typically several months. An open question for this problem is whether the optimum is actually a periodic solution. In order to gain insight on this particular point, we do not enforce here periodicity conditions. A second difference from the previous optimization is that we restrict the initial conditions to more realistic values, with $y(0) \in[0.05,0.25], s(0) \in[0.5,5]$ and $x(0) \in[0.5,3]$. We draw on Fig.7 the solutions for a time interval of 300 days (other timeframes give similar structures). The number of time steps has been increased in accordance with the larger timeframe (namely 15000).
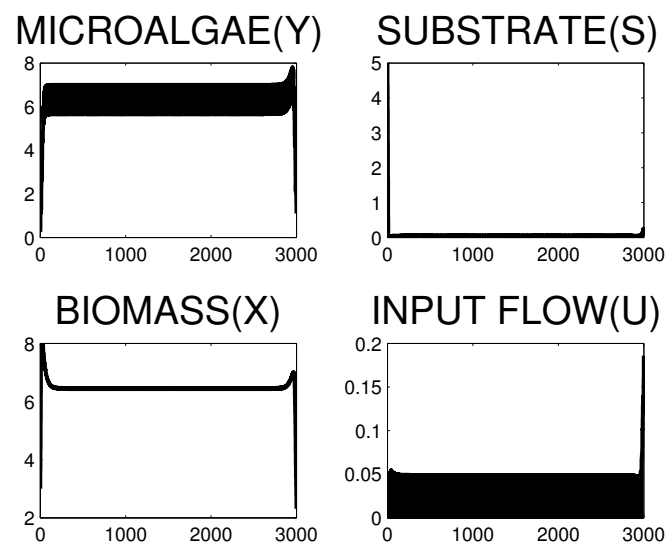

Figure 7: Optimization (non-periodic) over 300 days.

We observe that the initial conditions are always at their upper bound, i.e. $\zeta_{0}=(0.25,5,3)$, which means that the reactors are initially filled with as much algae, substrate and biomass as possible. Then, the overall structure of the solution is always divided into three distinct phases. During the first few days, a growth phase where both reactors are in batch mode $(u=0)$ and the concentration of microalgae and biomass increase, until they reach the values corresponding to the previously studied optimal 1-day cycle. Then, the bulk of the timeframe is filled with a sequence of periodic cycles, identical to the one optimized in 4.3.1. We recall that in this section, we did not enforce any periodicity conditions on the optimal control problem. Finally, the last few days show an increased input flow that gives a slight increase in production, at the expense of washing out the reactors (meaning the concentrations of algae and biomass decrease strongly). This last part is due to the absence of final conditions, and should probably be neglected for the actual operation of the reactor. 
We can also compare the full optimization over 300 days to the strategy that simply applies each day the control we found when optimizing a periodic 1-day cycle. Taking the same initial conditions $\zeta_{0}=(0.25,5,3)$, we observe that the attraction property holds and the trajectories for $(y, s, x)$ tend to the periodic ones, see Fig.8. The value of the cost function is also extremely close, at 274.8476 versus 277.3360 for the full optimization (see Table 2).

We see that the repetition of the 1-day periodic optimal process produces a cost which is close to the optimal one over a period of 300 days. Hence, we believe that repeating this cycle produces an accurate approximation of the optimal cost over a large timeframe. This is confirmed by choosing other timeframes.
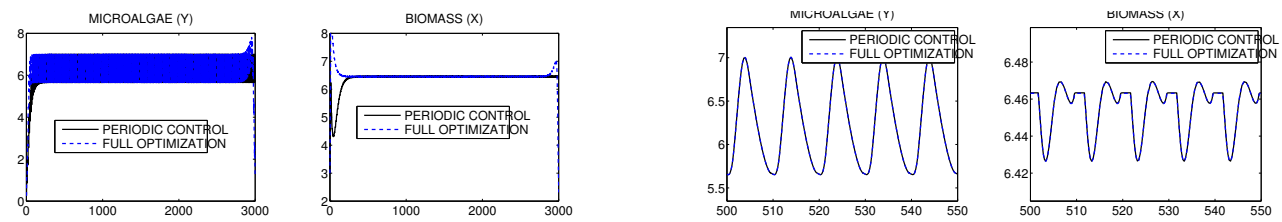

Figure 8: 1-day periodic control versus full optimization over 300 days (zoom on the right).

Table 2: Comparison of the cost function for several optimizations (volume ratio $\beta=1$ ).

\begin{tabular}{|l|r|r|r|}
\hline Optimization type & $t_{f}(n$ days $)$ & Production $J_{n}$ & $J_{n} / t_{f}$ \\
\hline 1-day periodic, free initial conditions $\zeta_{0}$ & 1 & 0.921 & 0.921 \\
Full optimization, $\zeta_{0}=(0.25,5,3)$ & 300 & 277.336 & 0.925 \\
Fixed 1-day periodic control, $\zeta_{0}=(0.25,5,3)$ & 300 & 274.848 & 0.916 \\
\hline
\end{tabular}

\subsection{Optimal volume ratio}

Until now, we assumed that the volume ratio of the two reactors was fixed to $\beta=1$. In this last series of simulations, we optimize this parameter in addition to the whole trajectory (state and control). We chose to impose $\beta \in[0.1,10]$ for the optimization.

\subsubsection{Optimal 1-day periodic strategy with free volume ratio}

We begin with optimizing a 1-day periodic cycle, as in 4.3.1, the only difference is that $\beta$ is now free. The obtained solution is shown on Fig.9, and the optimal volume ratio is $\beta_{1 d a y}^{*}=5.427$. Compared to the solution for $\beta=1$ (Fig.5), the concentration of algae, substrate and biomass are higher, as well as the input flow. A qualitative difference is that the two bang arcs with $u=0$ seem to have disappeared, the control being now fully singular. During these arcs, the production in the second reactor was stopped because the substrate was completely consumed by the biomass (i.e. $s=0$ ), indicating that the whole process was mainly limited by the microalgae culture. The higher volume ratio lifts this limitation and allows a better balance between the two processes, so the objective value is much better, at 1.430 versus 0.921 . In view of these simulations, we can conjecture that there exists a value of $\beta$ for which the optimal solution is fully singular. This point seems not obvious in view of the periodic constraint on the system.

\subsubsection{Optimization over $\mathrm{N}$ days with free volume ratio}

Next we move on to the optimization over a larger timeframe, while removing the periodicity conditions, and setting tighter bounds on the initial conditions. The simulations indicate that the solutions always saturate the upper bounds for the initial conditions, i.e. $\zeta_{0}=(0.25,5,3)$. Moreover, we observe the same three distinct phases as in the cases where $\beta=1$. We have a short initial growth phase, followed by a sequence of 1-day periodic cycles identical to the one found on Fig.9. This periodic phase represents most of the time interval, and on the last few days there is a phase with increased input flow that gives a bit more production at the expense of decreasing the concentrations of algae and biomass. We show on Fig.10 the solution for $t_{f}=300$ days, with $\beta_{300 \text { days }}^{*}=5.194$. 

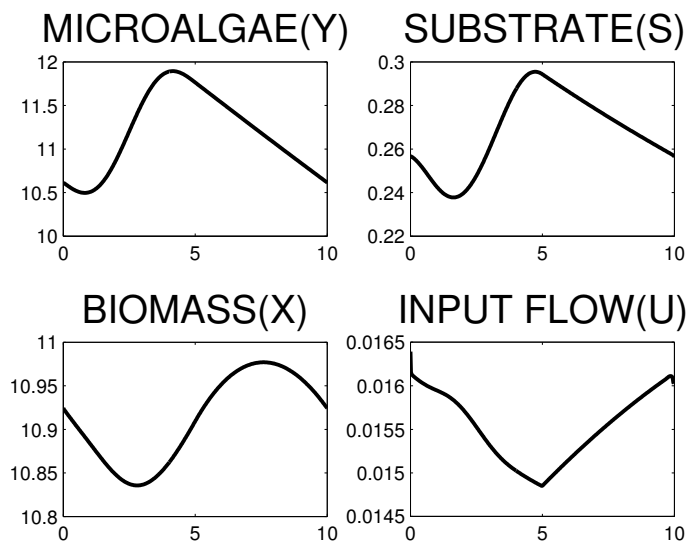

Figure 9: Solution with optimal volume ratio - 1-day periodic cycle.
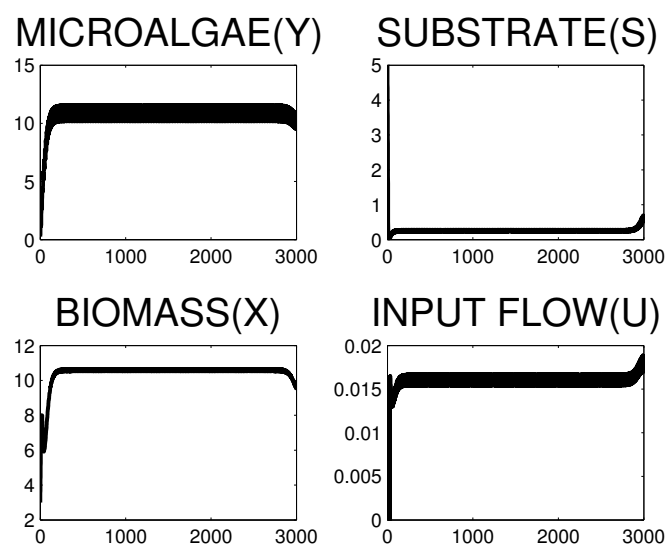

Figure 10: Solution with optimal volume ratio - 300 days.

\subsubsection{Link between $\mathrm{N}$-days optimization and 1-day periodic cycle}

When comparing the solutions for the optimal 1-day periodic cycle, and for the 300 days timeframe, we notice that the optimal volume ratio $\beta *$ is not the same (5.427 vs 5.194). This seems annoying at first, however numerical simulations indicate that while the optimal ratio indeed varies depending on the final time, it seems to tend to a limit value that corresponds to the 1-day periodic case (see Fig.11 and Table 3). We observe a similar behavior for the production per day, the reason being the strong link between the 1-day periodic solution and the optimal strategies for longer timeframes. According to the simulations, the optimal strategy for optimizing over $N$ days mainly consists in repeating the optimal periodic cycle for 1 day. The initial growth phase to reach the corresponding concentrations of algae and biomass only take a few days, as well as the final washing out phase. For larger $N$, the long-term solution becomes closer to simply repeating $N$ times the 1-day optimal cycle, both in terms of average production per day, and of optimal design parameter. This property would be interesting for practical purposes, indicating that optimizing the 1-day periodic cycle may provide most of the significant data for the design and operation of the reactors.

\section{Conclusion}

This paper studies the optimization of the production of biogas by anaerobic digestion of microalgae, over long timeframes. Modeling the two coupled bioreactors for this system gives a three-dimensional optimal control problem in Lagrange form. Thanks to the theory of Kolmogorov periodic equations, we obtain the existence 

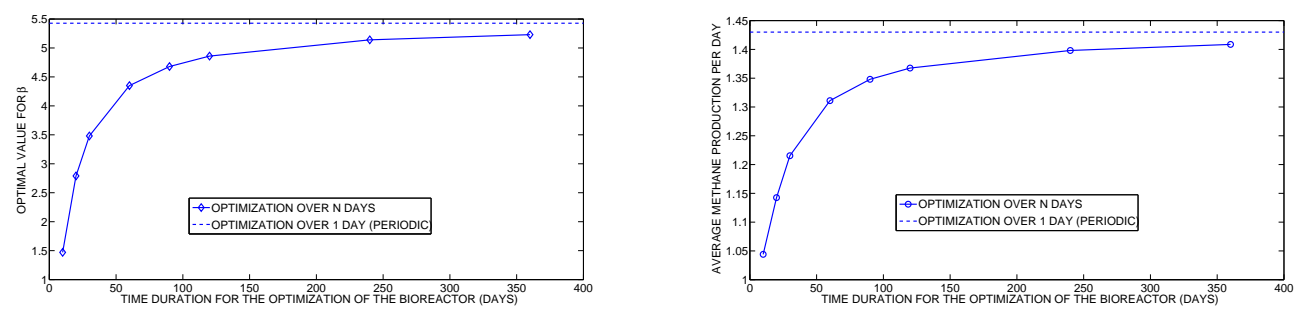

Figure 11: Optimal volume ratio and production per day.

Table 3: Comparison of the cost function for several optimizations (free volume ratio $\beta$ ).

\begin{tabular}{|l|r|r|r|r|}
\hline Optimization type & $t_{f}(n$ days $)$ & Production $J_{n}$ & $J_{n} / t_{f}$ & volume ratio $\beta$ \\
\hline Full optimization, $\zeta_{0}=(0.25,5,3)$ & 10 & 10.444 & 1.044 & 1.467 \\
Full optimization, $\zeta_{0}=(0.25,5,3)$ & 20 & 22.850 & 1.143 & 2.792 \\
Full optimization, $\zeta_{0}=(0.25,5,3)$ & 30 & 36.463 & 1.215 & 3.480 \\
Full optimization, $\zeta_{0}=(0.25,5,3)$ & 90 & 121.322 & 1.348 & 4.682 \\
Full optimization, $\zeta_{0}=(0.25,5,3)$ & 360 & 597.108 & 1.409 & 5.232 \\
\hline 1-day periodic, free initial conditions $\zeta_{0}$ & 1 & 1.430 & 1.430 & 5.427 \\
\hline
\end{tabular}

and attraction of periodic solutions over one day. By Pontryagin Maximum Principle, we can characterize extremal controls and compute singular arcs. A first series of numerical simulations using both direct and indirect methods illustrates the impact of the light model over the singular control. Then we compare the optimal strategy for a 1-day periodic cycle with the solutions for longer timeframes. Simulations suggest that the long-term optimal strategy consists in a initial growth phase in both reactors, followed by a sequence of periodic 1-day cycles. Finally we investigate the optimal volume ratio of the two reactors, with once again a strong link between 1-day periodic and long term solutions. In particular, it seems that there exists an optimal volume ratio that while depending on the timeframe for the optimization, tends to the optimal ratio for a 1-day periodic cycle. This property, if confirmed on more complex models, would be quite useful in the practical design of such coupled bioreactors. Similarly, the attraction of periodic solutions also deserves further investigation, as it may remove the practical need of a feedback controller.

\section{Appendix}

\subsection{Proof of Lemma 2.3}

Recall that $x(\cdot)$ and $\bar{x}(\cdot)$ satisfy the ODE:

$$
\dot{x}=x\left[\mu_{2}(\psi(t)-x)-\beta u(t)\right], \bar{x}=\bar{x}\left[\mu_{2}\left(\tilde{\psi}_{u}(t)-\bar{x}\right)-\beta u(t)\right], x(0)=\bar{x}(0)=x_{0} .
$$

Moreover $\psi$ and $\tilde{\psi}_{u}$ are uniformly bounded. In fact, one has that $\psi_{u}$ is bounded over $[0, T]$ and $\psi(t)-\tilde{\psi}_{u}(t) \rightarrow 0$ when $t$ goes to infinity. Hence, $x$ and $\tilde{x}_{u}$ are also uniformly bounded over $\mathbb{R}_{+}$. Now, set $z:=x-\bar{x}$ which satisfies $z(0)=0$ and notice that $s(t) \geq 0$. So, as $\mu_{2}^{\prime}$ is bounded by a positive constant over $\mathbb{R}_{+}$, the function

$$
t \longmapsto \eta_{1}(t):=x(t) \mu_{2}(\psi(t)-x(t))-x(t) \mu_{2}\left(\tilde{\psi}_{u}(t)-x(t)\right)
$$

goes to zero when $t$ goes to infinity. Using the fact that $\zeta \longmapsto \zeta \mu_{2}\left(\tilde{\psi}_{u}(t)-\zeta\right)$ is concave over $\left[0, \tilde{\psi}_{u}(t)\right]$, we obtain the inequality:

$$
\dot{z}(t) \leq \eta_{1}(t)-\beta u(t) z(t)+z(t)\left[\mu_{2}\left(\tilde{\psi}_{u}(t)-\bar{x}(t)\right)-\bar{x}(t) \mu_{2}^{\prime}\left(\tilde{\psi}_{u}(t)-\bar{x}(t)\right)\right], \quad t \geq 0 .
$$

Now using the boundedness of $x$ and $\bar{x}$ together with the fact that $\bar{x}(t)-\tilde{x}_{u}(t)$ goes to zero when $t$ goes to infinity, we can show that there exists a function $\eta: \mathbb{R}_{+} \rightarrow \mathbb{R}$ which goes to zero at infinity and such that the following inequality holds:

$$
\dot{z}(t) \leq \eta(t)+z(t)\left[-\beta u(t)+\mu_{2}\left(\tilde{\psi}_{u}(t)-\tilde{x}_{u}(t)\right)-\tilde{x}_{u}(t) \mu_{2}^{\prime}\left(\tilde{\psi}_{u}(t)-\tilde{x}_{u}(t)\right)\right], \quad t \geq 0 .
$$


Let us now define $\varphi:=-\beta u+\mu_{2}\left(\tilde{s}_{u}\right)-\tilde{x}_{u} \mu_{2}^{\prime}\left(\tilde{s}_{u}\right)$ which is $T$-periodic. The previous inequality becomes:

$$
\dot{z}(t) \leq \eta(t)+\varphi(t) z(t), t \geq 0 .
$$

Now, by integrating the ODE satisfied by $\tilde{x}_{u}$ over the period $[0, T]$, we obtain using the $T$-periodicity of $\tilde{x}_{u}$ :

$$
\int_{0}^{T}\left[\mu_{2}\left(\tilde{s}_{u}(t)\right)-\beta u(t)\right] d t=0 .
$$

Therefore, if we set $\delta:=\int_{0}^{T} \varphi(t) d t<0$, we can show easily that when $t$ goes to infinity, we have:

$$
\frac{1}{t} \int_{0}^{t} \varphi(s) d s \rightarrow \delta<0
$$

Combining (5.1),(5.2) and the fact that $\eta$ goes to zero, we can show that $z$ goes to zero at infinity (see e.g. $[17])$. This concludes the proof.

\subsection{Computation of the singular arc}

We provide in this section the expression of singular controls. The expression below are obtained by differentiating $\dot{\phi}$ w.r.t. $t$ and have been verified using a symbolic Software. If $\mu$ is given by (1.2), the differentiation is made on both intervals $[0, \bar{T})$ and $(\bar{T}, T])$. We start by the case where $t \longmapsto \mu(t)$ is constant. For convenience, we define $m_{1}, m_{2}, m_{3}$ as:

$$
\left\{\begin{array}{l}
m_{1}:=\beta(\gamma y-s)\left(\lambda_{2}-\lambda_{3}\right) x \mu_{2}^{\prime}(s), \\
m_{2}:=\mu_{2}(s) x \\
m_{3}:=y\left[-\lambda_{1} y \frac{\mu(t)}{(1+y)^{2}}+\lambda_{2} \beta \gamma\left(\frac{\mu(t)}{1+y}-r\right)\right] .
\end{array}\right.
$$

By differentiating w.r.t. $t$, we obtain:

$$
\begin{aligned}
& \dot{m}_{1}=x \beta\left(\lambda_{2}-\lambda_{3}\right)\left[(\gamma y-s)\left\{x\left[\mu_{2}^{\prime}(s)^{2}-\mu_{2}(s) \mu_{2}^{\prime \prime}(s)\right]+\mu_{2}^{\prime \prime}(s) \beta(\gamma y-s) u-\beta \mu_{2}^{\prime}(s) u\right\}\right. \\
& \left.+\mu_{2}^{\prime}(s)\left\{\gamma y\left[\frac{\mu(t)}{1+y}-(r+u)\right]+\mu_{2}(s) x\right\}\right]+x \beta \mu_{2}^{\prime}(s)(\gamma y-s) u, \\
& \dot{m}_{2}=\left(\mu_{2}(s)-u \beta\right) x \mu_{2}(s)+x \mu_{2}^{\prime}(s)\left[-\mu_{2}(s) x+u \beta(\gamma y-s)\right], \\
& \dot{m_{3}}=y\left\{\beta \gamma\left(\frac{\mu(t)}{1+y}-r\right)\left[x\left(\lambda_{2}-\lambda_{3}\right) \mu_{2}^{\prime}(s)+u \lambda_{2} \beta\right]\right\}+y\left[\frac{\mu(t)}{1+y}-(r+u)\right] \times \\
& {\left[-\lambda_{1} y \frac{\mu(t)}{(1+y)^{2}}+\lambda_{2} \beta \gamma\left(\frac{\mu(t)}{1+y}-r\right)\right]-y\left(\frac{\beta \gamma \mu(t)}{(1+y)^{2}} \lambda_{2}+\frac{\mu(t)(1-y)}{(1+y)^{3}} \lambda_{1}\right)\left[\frac{\mu(t) y}{1+y}-(r+u) y\right]} \\
& -y\left\{\frac{y \mu(t)}{(1+y)^{2}}\left(\left[-\frac{\mu(t)}{(1+y)^{2}}+r+u\right] \lambda_{1}-\lambda_{2} \beta \gamma u\right)\right\} \text {. }
\end{aligned}
$$

Therefore: $\ddot{\phi}(t)=\dot{m}_{1}+\dot{m}_{2}+\dot{m}_{3}$, and we get:

$$
\begin{aligned}
A_{\mu}(z, \lambda)= & x \mu_{2}(s)\left(\mu_{2}(s)-x \mu_{2}^{\prime}(s)\right)+x \beta\left(\lambda_{2}-\lambda_{3}\right)\left[x(\gamma y-s)\left(\mu_{2}^{\prime}(s)^{2}-\mu_{2}(s) \mu_{2}^{\prime \prime}(s)\right)\right] \\
& -\frac{y^{2} \mu(t)}{(1+y)^{2}}\left[-\frac{\mu(t)}{(1+y)^{2}}+r\right] \lambda_{1}+x \beta\left(\lambda_{2}-\lambda_{3}\right) \mu_{2}^{\prime}(s)\left(\gamma y\left(\frac{\mu(t)}{1+y}-r\right)+\mu_{2}(s) x\right) \\
& +y\left[\frac{\mu(t)}{1+y}-r\right]\left[-\lambda_{1} y \frac{\mu(t)}{(1+y)^{2}}+\lambda_{2} \beta \gamma\left(\frac{\mu(t)}{1+y}-r\right)\right] \\
& +y \beta \gamma\left(\frac{\mu(t)}{1+y}-r\right) x\left(\lambda_{2}-\lambda_{3}\right) \mu_{2}^{\prime}(s)-y\left(\frac{\beta \gamma \mu(t)}{(1+y)^{2}} \lambda_{2}+\frac{\mu(t)(1-y)}{(1+y)^{3}} \lambda_{1}\right)\left[\frac{\mu(t) y}{1+y}-r y\right],
\end{aligned}
$$




$$
\begin{aligned}
B_{\mu}(z, \lambda) & =x \beta\left[-\mu_{2}(s)+\mu_{2}^{\prime}(s)(\gamma y-s)\right]+x \beta \mu_{2}^{\prime}(s)(\gamma y-s)+x \beta^{2}\left(\lambda_{2}-\lambda_{3}\right)(\gamma y-s) \times \\
& {\left[\mu_{2}^{\prime \prime}(s)(\gamma y-s)-\mu_{2}^{\prime}(s)\right]-x \beta\left(\lambda_{2}-\lambda_{3}\right) \mu_{2}^{\prime}(s) \gamma y-y\left[-\lambda_{1} y \frac{\mu(t)}{(1+y)^{2}}+\lambda_{2} \beta \gamma\left(\frac{\mu(t)}{1+y}-r\right)\right] } \\
& +y \beta^{2} \lambda_{2} \gamma\left[\frac{\mu(t)}{1+y}-r\right]+y^{2}\left(\frac{\beta \gamma \mu(t)}{(1+y)^{2}} \lambda_{2}+\frac{\mu(t)(1-y)}{(1+y)^{3}} \lambda_{1}\right)-\frac{\mu(t) y^{2}}{(1+y)^{2}}\left(\lambda_{1}-\lambda_{2} \beta \gamma\right) .
\end{aligned}
$$

In the case where $\mu$ is differentiable a.e., we must take into account the derivative of $\mu$ in $m_{3}$. The term $A_{\mu}$ is replaced by $\tilde{A}_{\mu}$ where:

$$
\tilde{A}_{\mu}(z, \lambda):=A_{\mu}(z, \lambda)+y\left[\frac{-\lambda_{1} y}{(1+y)^{2}}+\frac{\lambda_{2} \beta \gamma}{1+y}\right] \mu^{\prime}(t) .
$$

Thus, the general expression of $\ddot{\phi}$ is: $\ddot{\phi}(t)=\tilde{A}_{\mu}(z(t), \lambda(t))+u(t) B_{\mu}(z(t), \lambda(t))$.

\subsection{Discussion of the optimal solution for the discontinuous light model}

One interesting point raised by the numerical simulations with the discontinuous light model (that is with $\left.\mu=\mu_{0}\right)$ is the following. Does an optimal trajectory cross the singularity $t=\bar{T}$ with a singular arc? More precisely, we would like to know if an optimal trajectory contains a singular arc on some time interval $\left[t_{1}, t_{2}\right]$, where $0<t_{1}<\bar{T}<t_{2}<T$. In view of the necessary conditions provided by the Pontryagin Maximum Principle, we believe that this situation cannot occur. As a consequence, the trajectory either jumps to an arc $B_{+}$or $B_{-}$in a neighborhood of the discontinuity time $t=\bar{T}$ in order to connect the two singular arcs on each phase. This leads to the structure $B^{-} S B^{+} S B^{-}$as in section 4.2.1.

In order to make this issue more precise, let us fix some notations. System (1.3) can be written:

$$
\dot{z}=f_{i}(z)+u g(z), i=1,2,
$$

where $z=(y, s, x)$ and $f_{i}, g$ are the vector fields defining the dynamics on $[0, \bar{T}]$ and $[\bar{T}, T]$. Also, we set $\ell(z):=\mu(s) x$ as the integrand of $J_{1}(u)$. Recall that for an optimal trajectory, the Hamiltonian is constant on each phase (as the system is autonomous on $[0, \bar{T}]$ and $[\bar{T}, T]$ ). The Lie bracket between $f_{i}$ and $g$ is denoted by $\left[f_{i}, g\right]$, see $[9]$.

Proposition 5.1. If a singular arc crosses $t=\bar{T}$, then the adjoint vector $\lambda(\bar{T})$ at time $t=\bar{T}$ must satisfy the five conditions:

$$
\left\{\begin{array}{l}
\lambda(\bar{T}) \cdot g(z(\bar{T}))=0 \\
\lambda(\bar{T}) \cdot f_{1}(z(\bar{T}))=H_{1} \\
\lambda(\bar{T}) \cdot f_{2}(z(\bar{T}))=H_{2} \\
\lambda(\bar{T}) \cdot\left[f_{1}, g\right](z(\bar{T}))=-\nabla \ell(z(\bar{T})) \cdot g(z(\bar{T})) \\
\lambda(\bar{T}) \cdot\left[f_{2}, g\right](z(\bar{T}))=-\nabla \ell(z(\bar{T})) \cdot g(z(\bar{T})) .
\end{array}\right.
$$

where $H_{i}, i=1,2$ is the Hamiltonian value on each phase.

Proof. The first condition of (5.3) means that the switching function $\phi$ satisfies $\phi(\bar{T})=0$ (consequence of the singular arc). The second and third conditions of (5.3) follow directly from the conservation of the Hamiltonian on each phase. Now, following [7], we have by a direct computation: $\dot{\phi}(t)=\left[f_{i}, g\right](z(t))+\nabla \ell(z(t)) \cdot g(z(t))$. We obtain the two last conditions by using the fact that $\dot{\phi}(t)=0$ on each phase.

If these five linear conditions on $\lambda(\bar{T})$ are not satisfied, the system (5.3) has no solution, and a singular arc cannot cross the discontinuity time. We have checked that condition (5.3) is not satisfied by the adjoint vector which was approximated by Lagrange multiplier (see section 4.1). This explains the existence of an additional Bang arc at the discontinuity point in order to connect the singular arcs on each phase. We believe that condition (5.3) is generically not satisfied and that consequently singular arc cannot occur in a neighborhood of the discontinuity $\bar{T}$.

\section{Acknowledgment}

This work was supported by Programa de Financiamiento Basal from the Center of Mathematical Modeling, Universidad de Chile and was developed in the context of DYMECOS INRIA associated team and of 
the program Stic-AmSud MOMARE. The first author thanks INRIA for providing him a one year research opportunity at INRA-INRIA project MODEMIC. The last author thanks the CIRIC (Communication and Information Research and Innovation Center) for providing him a research opportunity.

\section{References}

[1] Duran E, Isambert A, Joannis-Cassan C, Spolaore P. Commercial applications of microalgae. J. of bioscience and bioengineering 2006; 101:87-96.

[2] Barbosa M, Wijffels R. An outlook on microalgal biofuels. Science 2010; 329:796-799.

[3] Bernard O, Bernet N, Sialve B. Anaerobic digestion of microalgae as a necessary step to make microalgal biodiesel sustainable. Biotechnology Advances 2009; 27:409-416.

[4] Akhmetzhanov A, Bernard O, Grognard F, Masci P. Optimization of a photobioreactor biomass production using natural light. 49th CDC conference, 2010.

[5] Smith HL, Waltman P. The Theory of the Chemostat, Dynamics of Microbial Competition, Cambridge Studies in Mathematical Biology, vol. 13. Cambridge University Press: Cambridge, 1995, doi: 10.1017/CBO9780511530043.

[6] Budman H, Jervis E, Silveston P. Forced modulation of biological processes: A review. Chemical Engineering Science 2008; 63:5089-5105.

[7] Bonnard B, Chyba M. Singular Trajectories and their role in Control Theory, vol. 40. Springer SMAI, 2002.

[8] Schattler H, Ledzewicz U. Geometric Optimal Control. Springer, 2012.

[9] Trélat E. Contrôle optimal, théorie et applications. Vuibert, 2005.

[10] Bonnans F, Grelard V, Martinon P. Bocop, the optimal control solver, open source toolbox for optimal control problems 2011; URL http://bocop.org.

[11] Martinon P. Numerical resolution of optimal control problems by a piecewise linear continuation method. $\mathrm{PhD}$ thesis INP Toulouse, 2005.

[12] Kolmogorov A. Sulla teoria di volterra della lotta per l'esistenza. Giornale Istituto Ital. Attuari 1936; 7:74-80.

[13] Zanolin F. Continuation theorems for the periodic problem via the translation operator. Rend. Sem. Mat. Univ. Pol. Torino 1996; 54(1).

[14] Bastin G, Dochain D. On-line estimation and adaptive control of bioreactors. Elsevier: New York, 1990.

[15] Dochain D, Rapaport A. Minimal time control of fed-batch processes with growth functions having several maxima. IEEE Trans. Automat. Control 2011; 56(11):2671-2676, doi:10.1109/TAC.2011.2159424.

[16] Wolkowicz G, Zhao X. N-species competition in a periodic chemostat. Differential Integral Equations 1998; 11(3):465-491.

[17] Zhao X. Dynamical systems in population biology. Springer-Verlag, 2003.

[18] Bayen T, Mairet F, Sebbah M. Optimal control problem connected to a one-dimensional kolmogorov equation. preprint 2013; URL http://hal.archives-ouvertes.fr/hal-00855054.

[19] Cesari L. Optimization-Theory and Applications, Problems with Ordinary Differential Equations, Applications of Mathematics (New York), vol. 17. Springer-Verlag: New York, 1983.

[20] Pontryagin LS, Boltyanskii VG, Gamkrelidze RV, Mishchenko EF. The Mathematical Theory of Optimal Processes. Translated by D. E. Brown, A Pergamon Press Book. The Macmillan Co., New York, 1964. 
[21] Robbins HM. A generalized Legendre-Clebsch condition for the singular cases of optimal control. IBM J. of Research and Development 1967; 11:361-372.

[22] Betts J. Practical methods for optimal control using nonlinear programming. Society for Industrial and Applied Mathematics (SIAM): Philadelphia, 2001.

[23] Nocedal J, Wright S. Numerical optimization. Springer-Verlag: New-York, 1999.

[24] Pesch H. A practical guide to the solution of real-life optimal control problems. Control and Cybernetics 1994; 23:7-60.

[25] Aronna M, Bonnans F, Martinon P. A shooting algorithm for optimal control problems with singular arcs. J. Optim. Theory Appl. 2013; 158(2):419-459. 